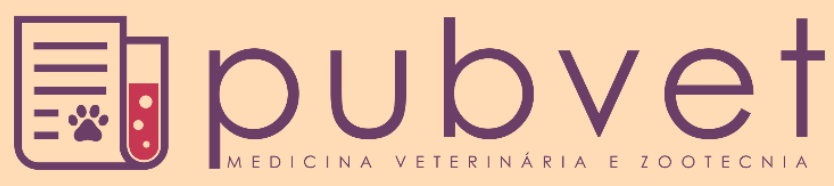

https://doi.org/10.31533/pubvet.v15n05a816.1-6

\title{
Tratamento com terapia neural em cão com discopatia: Relato de caso
}

\author{
Bruna Aparecida Lima Gonçalves ${ }^{1 *} \bullet \mathbb{D}$, Leonardo Rocha Vianna ${ }^{1} \mathbb{D}$, Ana Cristina Ribeiro \\ Mendes $^{2}$, Clara Angelina de Almeida ${ }^{3 \bullet}$ \\ ${ }^{I}$ Medico Veterinário. Mestre pela Universidade Federal de Minas Gerais. Homeopata, Acupunturista, Terapeuta Neural. \\ ${ }^{2}$ Professora da Pontifícia Universidade Católica de Minas Gerais, Departamento de Medicina Veterinária, Betim-MG Brasil. \\ ${ }^{3}$ Graduação Medicina Veterinária pela Pontifícia Universidade Católica de Minas Gerais Betim-MG Brasil. \\ *Autor para correspondência: E-mail: bruna_alg@yahoo.com.br
}

Resumo. A Terapia neural é um tipo de tratamento que baseado no histórico de vida do paciente se realiza aplicações de anestésicos locais em baixas concentrações, como o cloridrato de procaína ou lidocaína, com o objetivo de autorregulação corporal e autocura do organismo. No Brasil ainda é pouco conhecida, mas é difundida em países como Alemanha, Argentina, Canadá, Colômbia, Espanha, Suíça. Pode tratar inúmeras afecções incluindo a doença do disco intervertebral. Com esse estudo objetivou-se relatar o caso de uma cadela que apresentava incoordenação motora, perda da função dos membros pélvicos.

Palavras-chave: Analgesia, anti-inflamatório, cloridrato de procaína, doença do disco intervertebral, qualidade de vida

\section{Treatment with neural therapy in a dog with thoracolumbar discopathy: Case report}

\begin{abstract}
Neural Therapy is a type of treatment that based on the patient's life history, local anesthetics are applied in low concentrations, such as procaine hydrochloride or lidocaine, with the objective of body self-regulation and self-healing of the organism. In Brazil, it is still a little unknown, but extremely widespread in countries like Germany, Argentina, Canada, Colombia, Spain, Switzerland. Various conditions such as intervertebral disc diseases can be treated. The aim of this study was to report the case of a female dog that presented motor incoordination and loss of pelvic hind limb function.
\end{abstract}

Keywords: Analgesia, anti-inflammatory, procaine hydrochloride, intervertebral disc disease, quality of life

\section{Tratamiento con terapia neural en un perro con discopatía toracolumbar: Reporte de caso}

Resumen. La terapia neural es un tipo de tratamiento que, en base a la historia de vida del paciente, utiliza anestésicos locales en bajas concentraciones, como el clorhidrato de procaína o la lidocaína, con el objetivo de autorregulación corporal y autocuración del organismo. En Brasil aún es poco conocido, pero está muy extendido en países como Alemania, Argentina, Canadá, Colombia, España, Suiza. Puede tratar numerosas afecciones, incluida la enfermedad del disco intervertebral. El objetivo de este estudio fue reportar el caso de una perra que presentó descoordinación motora, pérdida de función de los miembros pélvicos.

Palabras clave: Analgesia, antinflamatorio, clorhidrato de procaína, enfermedad del disco intervertebral, calidad de vida 


\section{Introdução}

A terapia neural (TN) é uma terapia de auto regulação, que atua diretamente sobre o sistema nervoso, reorganizando o funcionamento, corrigindo os transtornos causados no sistema elétrico celular e tissular (Klinghardt, 1993; Vianna \& Gonçalves, 2016). A técnica da TN baseia-se na aplicação de anestésicos locais diluídos em baixas concentrações em pontos específicos do paciente que podem ser pontos de acupuntura, cicatrizes toxicas, campos interferentes, via intravenosa, intraperitoneal, em gânglios, dentre outros (Gonçalves et al., 2019).

A terapia neural assim como a acupuntura possuem diversos efeitos fisiológicos em todo o organismo. Ambas são minimamente invasivas. São tratamentos regulatórios que buscam uma abordagem do todo (Steiis, 2001; Weinschenk, 2012).

Sabe se da existência dos canais de energia e suas relações com os órgãos internos (White \& Ernst, 2004). Segundo Molano et al. (2014) a medicina chinesa descreve há muitos anos os canais de energia, 235 acupontos correlacionam com zonas head. De acordo com Head em 1898 algumas enfermidades viscerais são refletidas em regiões sensíveis na pele.

Os acupontos estão localizados em áreas com menor resistência elétrica no corpo comparado com a pele ao redor (Draehmpaehl \& Zohmann, 1997). Estes acupontos apresentam maior aporte sanguíneo, nervoso e podem estar próximas a tendões, cápsulas articulares e periósteos ( $\mathrm{Wu}, 1990)$. O tratamento não segue um padrão predeterminado, cada paciente recebe um tratamento individualizado (Gonçalves et al., 2019).

Em 1902 Spiess observa os efeitos curativos dos anestésicos locais, em 1905 Einhorn descobre a procaína, o primeiro anestésico local sintético, em 1906 Wischnewsky afirma que a procaína possui ação anti-inflamatória. Em 1925, Leriche aplicou a procaína no gânglio estrelado com finalidades terapêuticas e os irmãos alemães Ferdinand e Walter Huneke batizaram a terapia dos anestésicos locais como terapia neural (Castro, 2011). Em 1936, Speransky, médico russo escreveu sobre a participação do sistema nervoso nos diferentes processos patológicos (Fischer, 2006). E em 1970, Gérman Duque, médico colombiano formado na Alemanha introduz a terapia neural na América Latina (Vianna \& Gonçalves, 2017).

Segundo Cassuto et al. (2006), os anestésicos locais possuem ação anti-inflamatória e antimicrobiana têm um potencial de 290 milivolts e efeito repolarizante, ou seja, a sua aplicação faz com que a célula repolarize e estabilize seu potencial de membrana (Burrel, 2008).

De acordo com Klinghardt (1993) a terapia neural é um tratamento que pode ser utilizado em quadros de dores, as aplicações podem ser em gânglios, próximo a nervos, cicatrizes, pontos gatilhos e outras áreas corporais. Sabe se que $70 \%$ dos pontos gatilhos são pontos de acupuntura regulares, e todos os pontos de acupuntura podem ser estimulados com a Terapia Neural (Vianna \& Gonçalves, 2016). Em um estudo em cães com dermatite atópica utilizou a dose máxima de $7 \mathrm{mg} / \mathrm{kg}$ de cloridrato de procaína 0,7\% de forma intradermal (Bravo-Monsalvo et al., 2008).

Segundo Harris (2010) a dor é um problema mundial em humanos e é a primeira causa de incapacidade física, é um dos principais problemas de saúde pública, afeta a qualidade de vida gerando consequências no âmbito pessoal e familiar. A terapia neural é um tratamento que pode ser utilizado em enfermidades agudas e crônicas. Um estudo em pacientes com lombalgia inespecífica demonstrou a eficácia da Terapia Neural em pontos de acupuntura (Medrano García et al., 2011).

Quadros degenerativos do disco intervertebral de cães condrodistróficos são mais comuns devido a genética ou até mesmo as deficiências de apoio proporcionadas pela anatomia do animal, e a coluna vertebral compensa com mineralização, degeneração, protusão e/ou extrusão material do núcleo pulposo (Brisson, 2010). O tratamento varia de acordo com o grau de gravidade da lesão, este pode ser conservativo ou cirúrgico. O conservativo está associado ao repouso, analgésicos, relaxantes musculares, anti-inflamatórios esteroidais e não esteroidais, acupuntura e fisioterapia (Levine et al., 2007; Silveira et al., 2020).

O presente trabalho tem como objetivo relatar um caso de canino com discopatia, atendido no hospital veterinário da PUC Minas em Betim. 


\section{Relato de caso}

Uma cadela Shih-tzu, com idade de sete anos e cinco meses foi atendida no Centro de Estudos em Clínica e Cirurgia de animais (CECCA) em Betim. Apresentava marcha descoordenada, perda de tônus na musculatura dos membros pélvicos e sensibilidade na região toracolombar e lombar. Ao toque ou manuseio do animal, ela defecava e urinava. Não tinha histórico de traumas. Ao exame semiológico o animal se mostrou consciente; porém, tinha perda de propriocepção nos membros pélvicos esquerdo e direito, perda da sensibilidade superficial para os membros pélvicos, mas normal para membros torácicos, palpação dolorosa nas regiões toracolombar e lombar e perda de tônus muscular dos membros pélvicos.

Foi realizado radiografia nas posições latero lateral, com foco na região toracolombar e lombar (Figura 1). Pela imagem foi constatado a mineralização óssea no espaço intervertebral das torácicas T12 e T13, havia estreitamento do espaçamento intervertebral entre torácicas T10-T11-T12 e no segmento lombar entre as lombares L4-L5. Os tutores tinham poucos recursos financeiros para realização de outros exames de imagem.

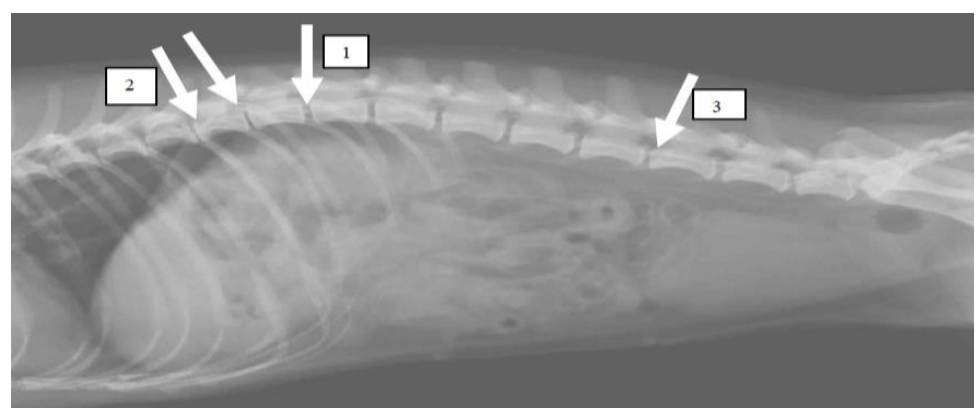

Figura 1. Radiografia na posição látero-lateral mostrando na seta 1 a mineralização entre a T12 e T13, nas seta 2 mostrando a redução dos espaços vertebrais entre T10, T11 e T12 e na seta 3 a redução nos espaços vertebrais L4 com L5. (Centro de imagens do CECCA).

Após a consulta com o clínico o paciente foi encaminhado para a Terapia Neural iniciando o tratamento no dia seguinte. Foi iniciada então a TN com uso de cloridrato de procaína a $0,7 \%$ nos seguintes pontos de acupuntura, B 11, B 18, B 21, B 23; B 25, B 60, R 3; E 36 e BAIHUI (Espaço Lombo sacro) (Figura 2).

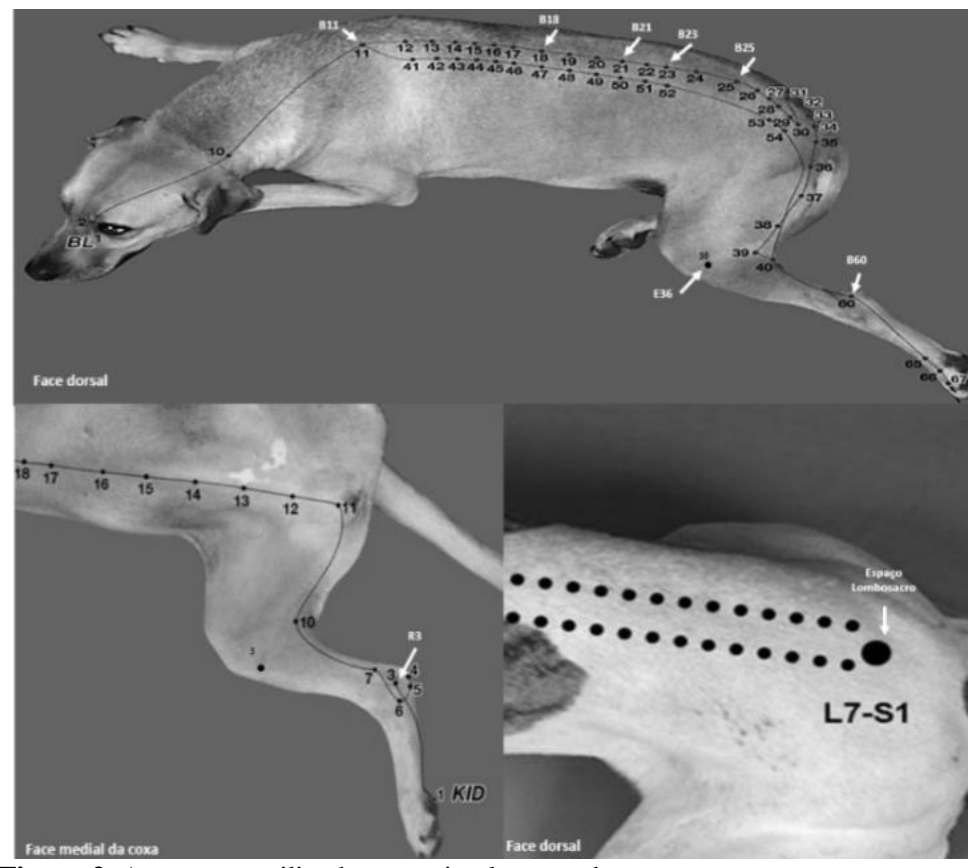

Figura 2. Acupontos utilizados no animal mostrados por setas. 
Após a primeira sessão a tutora relatou que o animal apresentou melhor apoio dos membros pélvicos, da marcha e redução da dor. As três primeiras sessões foram semanais, quando a médica veterinária acupunturista e terapeuta neural optou pelo espaçamento das sessões, após melhora do quadro e evolução do paciente.

Por intercorrências da tutora em levar o animal a quarta sessão só ocorreu após 30 dias da terceira e após avaliação, observou se que houve melhora significativa da sensibilidade dolorosa, normalidade dos reflexos e propriocepção, porém o animal ainda apresentava diferença de tônus muscular do membro pélvico direito para o membro pélvico esquerdo. Após seis sessões realizadas o animal apresentou recuperação clínica.

\section{Discussão}

A doença do disco intervertebral é uma degeneração do núcleo pulposo que pode levar à extrusão e/ou protusão discal nos espaços intervertebrais, sendo muito comum entre as vertebras torácicas T11T12 (Fossum, 2014). A mineralização do disco intervertebral é comum em cães condrodistróficos, o que aumenta a probabilidade de extrusão (Jensen et al., 2008). No caso relatado a cadela apresentava mineralização entre as vertebras torácicas T12 e T13 e redução de espaço intervertebral das torácicas T10-T11- T12 e lombares L4-L5. Por restrições financeiras não foi possível realizar outros exames de imagem. Segundo Santos et al. (2011) na radiografia simples a confirmação do diagnóstico fica limitada, mas diminuição do espaço intervertebral ou material calcificado dentro do canal vertebral são combatíveis com doença do disco intervertebral.

Pesquisas realizadas por McKee (2000) mostram que 90\% dos cães condrodistróficos apresentam: degenerações, herniações compressões e alterações ósseas como hemivértebra que podem afetar múltiplos discos intervertebrais. Os principais sinais clínicos observados nas discopatias toracolombares são perda de locomoção, dor abdominal, paresia dos membros pélvicos, podendo chegar à perda de percepção da dor profunda, incontinência urinária e fecal (Feldman, 1997). Alguns destes sintomas apresentados pelo animal deste relato.

Segundo Levine et al. (2007), pode-se observar melhora no quadro clínico com a administração de AINE's, quando comparados ao uso de corticoides, além da redução de recidivas. Em casos de dor crônica com déficits proprioceptivos mais graves deve haver descompressão cirúrgica. Segundo Peralta et al. (2011) a terapia neural diminui a dor dos pacientes e consequentemente o consumo de fármacos. E se mostrou eficiente em 280 pacientes com dores crônicas refratárias a tratamentos convencionais (Egli et al., 2015). De acordo com Cassuto et al. (2006) os anestésicos locais têm ação anti-inflamatória e apresentam ação repolarizante celular normalizando o potencial de membrana das células afetadas ajudando o organismo a retornar ao equilíbrio (Castro, 2011; Harris, 2010).

A escolha de tratamento do animal deste estudo foi a terapia neural acupuntural pela aplicação de cloridrato de procaína a $0,7 \%$ nos acupontos. A associação da terapia neural e da acupuntura faz com que os dois tipos de tratamento sejam potencializados (Braga \& Silva, 2012; Molano et al., 2014; Nozabieli et al., 2000; Scognamillo-Szabó \& Bechara, 2001).

É de vital importância a conscientização dos tutores no tratamento e ajustes no manejo em casa para evitar recidivas. $\mathrm{O}$ tratamento cirúrgico é a abordagem indicada para pacientes não responsivos ao manejo conservador, com episódios repetidos de dor e com déficits neurológicos progressivos, mas nesse caso o animal teve resposta ao tratamento (Brisson, 2010; Silveira et al., 2020). Sempre deve se levar em consideração a individualidade por meio do histórico de vida do paciente.

Com base nos resultados obtidos, a terapia neural pode ser um tratamento indicado para discopatia, visto que foi observada uma melhora clínica.

\section{Conclusão}

A doença do disco intervertebral é comum na clínica de pequenos animais, pode acometer cães e gatos de todas as idades. O animal teve a melhora dos sinais clínicos e diminuição do quadro de dor com $\mathrm{o}$ tratamento. $\mathrm{O}$ efeito anti-inflamatório dos anestésicos locais e o impulso neural terapêutico contribuíram para a melhora do paciente. O objetivo da terapia neural é a autorregulação corporal após 
o estímulo, para que este consiga retornar ao equilíbrio. Consequentemente melhorando a qualidade de vida ao paciente e todos em seu entorno.

\section{Referências}

Braga, N. S., \& Silva, A. R. C. (2012). Acupuntura como opção para analgesia em veterinária. PUBVET, 6, Art-1429.

Bravo-Monsalvo, A., Vázquez-Chagoyán, J., Gutiérrez, L., \& Sumano, H. (2008). Clinical efficacy of neural therapy for the treatment of atopic dermatitis in dogs. Acta Veterinaria Hungarica, 56(4), 459-469.

Brisson, B. A. (2010). Intervertebral disc disease in dogs. The Veterinary Clinics of North America. Small Animal Practice, 40(5), 829-858.

Burrel, S. E. (2008). Salud, enfermaria y terapia neural.

Cassuto, J., Sinclair, R., \& Bonderovic, M. (2006). Anti-inflammatory properties of local anesthetics and their present and potential clinical implications. Acta Anaesthesiologica Scandinavica, 50(3), 265-282. https://doi.org/10.1111/j.1399-6576.2006.00936.x.

Castro, R. A. (2011). Bases para la terapia neural em caninos y felinos. Dunken.

Draehmpaehl, D., \& Zohmann, A. (1997). Acupuntura no cão e no gato: princípios básicos e prática científica. Roca.

Egli, S., Pfister, M., Ludin, S. M., de la Vega, K. P., Busato, A., \& Fischer, L. (2015). Long-term results of therapeutic local anesthesia (neural therapy) in 280 referred refractory chronic pain patients. $B M C$ Complementary and Alternative Medicine, 15(200), 1-9. https://doi.org/10.1186/s12906-015-0735-z.

Feldman, E. C. (1997). Tratado de medicina interna veterinária. In Moléstias do cão e do gato (Vol. 3).

Fischer, L. (2006). Terapia neural según Huneke fundamentos técnicas, aplicación práctica. Hippokrates.

Fossum, T. W. (2014). Cirurgia de pequenos animais (4th ed., Vol. 1). Elsevier Brasil.

Gonçalves, B. A. L., Vianna, L. R., \& Andrade, C. C. (2019). Terapia Neural no tratamento do megaesôfago congênito em cão - relato de caso. PUBVET, 13(9), 1-6.

Harris, G. R. (2010). Effective treatment of chronic pain by the integration of neural therapy and prolotherapy. Journal of Prolotherapy, 2(2), 377-386.

Jensen, V. F., Beck, S., Christensen, K. A., \& Arnbjerg, J. (2008). Quantification of the association between intervertebral disk calcification and disk herniation in Dachshunds. Journal of the American Veterinary Medical Association, 233(7), 1090-1095. https://doi.org/10.2460/javma.233.7.1090.

Klinghardt, D. K. (1993). Neural therapy. Journal of Neurological and Orthopaedic Medicine and Surgery, 14, 109. https://doi.org/10.1136/bmj.2.5155.824-b.

Levine, J. M., Levine, G. J., Johnson, S. I., Kerwin, S. C., Hettlich, B. F., \& Fosgate, G. T. (2007). Evaluation of the success of medical management for presumptive thoracolumbar intervertebral disk herniation in dogs. Veterinary Surgery, 36(5), 482-491. https://doi.org/10.1111/j.1532950X.2007.00295.x.

McKee, M. (2000). Intervertebral disc disease in the dog 1. Pathophysiology and diagnosis. In Practice, 22(7), 355-369. https://doi.org/10.1136/inpract.22.8.458.

Medrano García, R., Varela Hernández, A., de la Torre Rosés, M., Mendoza Cisneros, R., \& Acosta Davison, Y. (2011). Resultados de la aplicación de la terapia neural en la lumbalgia inespecífica. Revista Archivo Médico de Camagüey, 15(1), 1-11.

Molano, M. L. B., Bonilla, L. B. P., Dussan, E. H. B., \& Londoño, C. A. V. (2014). Anatomo-functional correlation between head zones and acupuncture channels and points: a comparative analysis from the perspective of neural therapy. Evidence-Based Complementary and Alternative Medicine, 2014, $1-18$.

Nozabieli, A. J. L., Fregonesi, C. E. P. T., \& Fregonesi, D. A. (2000). Correlação dos canais de acupuntura com a neuroanatomia e a neurofisiologia. Arquivos de Ciências Da Saúde Da UNIPAR, 4(3), 263-268. 
Peralta, O. L., Rejón, A. R., Morales, D. P., Amores, A. G., Casajoana, D. V., \& Costa, K. P. V. (2011). Estudio de intervención sobre el dolor subagudo y crónico en atención primaria: una aproximación a la efectividad de la terapia neural. Atención Primaria, 43(11), 604-610. https://doi.org/10.1016/j.aprim.2010.10.004.

Santos, R. P., Mazzanti, A., Beckmann, D. V, Berté, L., Ripplinger, A., Neto, D. P., \& Baumhardt, R. (2011). Recuperação funcional em cães com doença do disco intervertebral toracolombar sem percepção à dor profunda: 37 casos (2002-2010). Pesquisa Veterinária Brasileira, 31(4), 345-349.

Scognamillo-Szabó, M. V. R., \& Bechara, G. H. (2001). Acupuntura: bases científicas e aplicações. Ciência Rural, 31(6), 1091-1099.

Silveira, S. D., Batschke, C. F., Minuzzo, T., Correa, F. L., \& Rorig, M. C. L. (2020). Hemilaminectomia como tratamento de discopatia toracolombar canina: Estudo retrospectivo e relato de caso. PUBVET, 14(4), 1-12. https://doi.org/10.31533/pubvet.v14n4a544.1-123.

Steiis, J. E. (2001). The neurophhysiologic basis od acupunture. In A. M. Schoen (Ed.), Veterinary acupunture: ancient art to moderne medicine (pp. 27-46). Mosby CO.

Vianna, L. R., \& Gonçalves, B. A. L. (2016). Nasce uma nova especialidade na Medicina Veterinaria Brasileira: Terapia Neural e Odontologia NeuroFocal. Revista Veterinária e Zootecnia, 130, 16-21.

Vianna, L. R., \& Gonçalves, B. A. L. (2017). Entenda a terapia neural. Revista CFMV, 74, 44-47.

Weinschenk, S. (2012). Neural therapy - a review of the therapeutic use of local anesthetics. Acupuncture and Related Therapies, 1(1): 5-9., 1(1), 5-9.

White, A., \& Ernst, E. (2004). A brief history of acupuncture. Rheumatology, 43(5), 662-663. https://doi.org/10.1093/rheumatology/keg005.

Wu, D.-Z. (1990). Acupuncture and neurophysiology. Clinical Neurology and Neurosurgery, 92(1), $13-$ 25. https://doi.org/Acupuncture and neurophisiology.

\section{Histórico do artigo:}

Recebido: 21 de novembro de 2020.

Aprovado: 19 de dezembro de 2020

Disponível online: 14 de abril de 2021 .
Licenciamento: Este artigoé publicado na modalidade Acesso Aberto sob a licença Creative Commons Atribuição 4.0 (CC-BY 4.0), a qual permite uso irrestrito, distribuição, reprodução em qualquer meio, desde que $o$ autore a fonte sejam devidamente creditados. 